\title{
O papel da vinculação aos pais e da regulação emocional no investimento esquemático na aparência em jovens adultos*
}

\author{
The Role of Parental Attachment and Emotional Regulation on \\ Schematic Investment in Appearance in Young Adults
}

\author{
Patrícia da Costa Claro $^{1}$ \\ Catarina Pinheiro Mota ${ }^{2}$ \\ ${ }^{1}$ Universidade de Trás-os-Montes e Alto Douro, Departamento de Educação e Psicologia,Portugal. \\ ${ }^{2}$ Universidade de Trás-os-Montes e Alto Douro, Departamento de Educação e \\ Psicologia e Centro de Psicologia da Universidade do Porto, Portugal.
}

\begin{abstract}
Resumo. Os vínculos afetivos que se estabelecem na infância podem ter efeitos no desenvolvimento emocional dos jovens. A vinculação segura pode potenciar a capacidade de regulação emocional e proporcionar uma imagem de si mais positiva, gerando comportamentos ajustados de investimento na aparência. A presente investigação objetivou analisar o papel da vinculação aos pais e da regulação emocional no investimento esquemático na aparência. Foram usados instrumentos de autorrelato numa amostra constituída por 948 jovens adultos (18-30 anos) de ambos os sexos. Evidenciou-se o papel significativo que a qualidade de vinculação aos pais e a regulação emocional assumem no desenvolvimento de um investimento ajustado na aparência. Verificou-se ainda o efeito mediador positivo das dificuldades de regulação emocional entre a inibição da exploração e individualidade mãe e a saliência auto-avaliativa.
\end{abstract}

Palavras-chave. Vinculação aos pais, investimento esquemático na aparência, regulação emocional, jovens adultos, mediador.

Abstract. The affective bonds that are established in childhood can have effects on the emotional development of young people. The secure attachment can potentiate the ability of emotional regulation, provide a more positive image of yourself and, therefore, adjusted investment behaviors in appearance. This study aims to analyze the role of parental attachment and the emotional regulation in the schematic investment in appearance. Self-report instruments were used on a sample that consisted of 948 young adults (18 and 30 years) of both genders. The study highlighted the significant role that the quality of parental attachment and emotional regulation take in developing an investment adjusted in appearance. The results showed the positive mediator effect of difficulties in emotional regulation between the inhibition of exploration and individuality mother and the selfevaluative salience.

Keywords. Parental attachment, schematic investment in appearance, emotional regulation, young adults, mediation.

*Esta investigação é parcialmente suportada pela FCT de acordo com o projeto PEst-C/PSI/UI0050/2011 e FEDER fundos do programa COMPETE inserido no projeto FCOMP-01-0124-FEDER-022714.

${ }^{1}$ Patrícia da Costa Claro. Universidade de Trás-os-Montes e Alto Douro - UTAD. Dirección Postal: Edifício das Ciências Humanas e Sociais - Polo I - UTAD, 5000-801 Vila Real, Portugal. E-mail: patyclaro_27@hotmail.com

${ }^{2}$ Catarina Pinheiro Mota. Universidade de Trás-os-Montes e Alto Douro (UTAD) e Centro de Psicologia da Universidade do Porto, Portugal. E-mail: catppmota@utad.pt

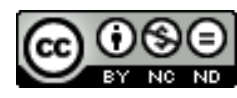

Esta obra está bajo una licencia de Creative Commons Reconocimiento-NoComercial-SinObraDerivada 4.0 Internacional. 


\section{Introdução}

Bowlby (1988) teorizou que a organização de vinculação estabelecida na infância pode servir como base para a forma como o indivíduo lida com stressores e regula as emoções na idade adulta. A teoria da vinculação desenvolvida por este autor conceptualizou a propensão dos seres humanos em estabelecer fortes vínculos afetivos com as figuras cuidadoras, decorrente da qualidade das interações com as mesmas (Bowlby, 1969). Das relações de proximidade e, consequentemente, das experiências e interações que crianças e jovens mantêm com as figuras significativas, constroem-se estilos de vinculação seguros ou inseguros que conduzem ao desenvolvimento dos modelos internos dinâmicos. Estes modelos integram mapas cognitivos, sentimentos, crenças, expectativas, basicamente representações que a criança constrói e tem sobre si própria e o seu comportamento, bem como dos outros e do mundo, e cuja complexificação tende a aumentar ao longo da vida (Bowlby, 1988). Uma característica particular é a capacidade de reorganização dos modelos e de adaptação às características dos novos períodos de desenvolvimento e contextos ao longo do ciclo de vida. Os modelos internos dinâmicos tornam-se mais positivos sob o ponto de vista da adaptação à medida que a procura de proximidade da criança vai sendo correspondida de forma satisfatória pelas figuras cuidadoras primárias, facilitando o desenvolvimento de uma base segura. A base segura assume especial relevância na abordagem da vinculação, integrando o sentimento de confiança em si, que se prende com uma elaboração precoce de correspondência afetiva por parte de uma figura de apoio e proteção acessível e disponível (Bowlby, 1988). O estabelecimento destes laços irá funcionar como fator protetor face a situações de risco, e como base segura para a criação de uma imagem positiva de si e dos outros, bem como a exploração do meio circundante (Ainsworth, 1989; Bowlby, 1969).

Deste modo, Bowlby (1969) aponta que a qualidade das relações de vinculação, ainda que não de forma determinante, podem servir de modelo para a construção de relações futuras ao longo da vida. A figura de vinculação capaz de satisfazer as necessidades de conforto e proteção da criança proporciona-lhe a construção de modelos internos marcados por confiança e valor próprio. A falta de confiança pode restringir à criança oportunidades para desenvolver competências, assim a vinculação insegura pode propiciar atraso no desenvolvimento cognitivo, falta de confiança em si e nos demais, podendo ainda haver problemas ao nível da regulação emocional (Malekpour, 2007).

Nesta perspetiva, de acordo com Bowlby (1973), os laços afetivos estabelecidos desde a infância poderão ter efeito na regulação e desenvolvimento emocional dos indivíduos ao longo da vida. A relação com as figuras significativas é a primeira relação emocional e a qualidade da mesma, poderá ditar a capacidade do individuo se autorregular emocionalmente. Assim, um estilo de vinculação inseguro poderá potenciar emoções negativas, como medo e ansiedade, restrição na expressão e compreensão de emoções, proporcionando, desta forma, maiores dificuldades de regulação emocional. Por outro lado, é esperado que indivíduos com tipo de vinculação segura, através de interações com as figuras cuidadoras, sejam capazes de expressar abertamente as suas emoções e aprendam maneiras eficazes para lidar com emoções negativas em situações ansiogénicas (Bowlby, 1973; Contreras \& Kerns, 2000). 
Bargh e Williams (2007), conceptualizaram a regulação emocional como o processo pelo qual os indivíduos, de forma consciente ou não consciente, modulam as suas emoções em resposta às exigências ambientais. As investigações na área da regulação emocional tiveram maior ênfase na infância especialmente nos processos de regulação emocional extrínseca, ou seja, aqueles que são desenvolvidos e construídos no vínculo com os outros (Borelli et al., 2017; Gross \& Thompson, 2006; Kim, Stifter, Philbrook, \& Teti, 2014; McMillen, Katz \& Claypool, 2014).

Moutsiana et al. (2014), apontam, num estudo realizado ao longo de 22 anos, o papel da vinculação na regulação de estados emocionais em jovens adultos. Nesta medida, 100 bebés que haviam sido submetidos à experiência de "situação estranha" (Ainsworth, 1969, para uma revisão), foram categorizados, de acordo com a resposta à experiência, como detendo uma vinculação segura ou insegura, sendo que 63 deles foram novamente analisados depois de 22 anos. A análise evidenciou que a vinculação insegura pode ter um efeito persistente sobre a regulação neural da emoção, com implicações para o ajustamento psicológico na idade adulta.

Desta forma, o estudo desta temática tem vindo a estender-se à adultícia, com base em que os processos de regulação emocional continuam em mudança e em desenvolvimento com a idade (Zimmerman \& Iwanski, 2014). Embora a relação entre vinculação aos pais e regulação emocional esteja documentada, faltam estudos que aprofundem a exploração de dificuldades específicas de regulação emocional em função da vinculação (GuzmánGonzález, Carrasco, Figueroa, Trabucco, \& Vilca, 2016). No contexto da investigação da regulação emocional em adultos e objetivando sistematizar as dificuldades neste grupo etário, Gratz e Roemer (2004), elaboraram um modelo em que concebem o processo de regulação emocional e as dificuldades associadas à mesma, não só a partir da modulação de afetos negativos e na execução de objetivos traçados, mas também no conhecimento, compreensão e aceitação das emoções e a possibilidade de aceder a estratégias de regulação percebidas como efetivas.

O estudo de Morel e Papouchis (2015), com 125 estudantes universitários, objetivando analisar o papel da vinculação na regulação emocional, corrobora a literatura, sugerindo que o estilo de vinculação parece associar-se com a capacidade do indivíduo em regular as emoções. Os resultados relataram que indivíduos com vinculação segura tinham menos dificuldade em regular as suas emoções. Evidenciou-se ainda que a vinculação insegura foi associada à falta de consciência emocional, limitação no uso de estratégias de regulação emocional e dificuldades em controlar os impulsos. A vinculação evitante associou-se à recusa de respostas emocionais e à falta de consciência e clareza emocional.

A qualidade de vinculação que os jovens mantêm com os pais, pode também ter um efeito na forma como estes percecionam as pressões sociais relativas ao corpo e constroem imagens do mesmo e de si mais saudáveis e positivas, uma vez que é no contexto desenvolvimental que se vivenciam as primeiras experiências sensoriais e corporais (Barbosa \& Costa, 2001). Indivíduos com vinculação segura, mostram-se mais confiantes, seguros, com maiores níveis de autoestima e autoaceitação no que diz respeito à sua imagem (Bowlby, 1969; Pietromonaco \& Barret, 2000). Por outro lado, a vinculação insegura, caracterizada por 
perceções negativas de si e dos outros, potencia sentimentos também mais negativos com o corpo, podendo haver maior suscetibilidade a comparações, avaliações e aceitação de outras pessoas e ainda a almejar compatibilidade com os protótipos de beleza (Bowlby, 1969; Park \& Beaudet, 2007). Para Troisi et al. (2006), a insatisfação corporal, proveniente de uma vinculação insegura, onde o indivíduo desloca a atenção para o seu corpo, pode estar associada ao medo da rejeição.

Para Barbosa, Matos e Costa (2011), a imagem corporal é uma "construção biopsicossocial", baseada nas experiências vividas ao longo do tempo. Cash (2008) encara-a mais do que uma imagem mental que se possui acerca da aparência. Para o autor, a imagem corporal consiste também na relação pessoal com o próprio corpo, englobando as perceções, crenças, pensamentos, sentimentos e ações que dizem respeito à aparência física de um indivíduo. A componente cognitiva ou de investimento na aparência (ligada às crenças e pensamentos acerca da imagem corporal) desempenha um papel central, segundo o autor, no conceito de imagem corporal. $\mathrm{O}$ investimento na aparência, abrange o conceito de esquemas de aparência, surgindo o termo investimento esquemático na aparência. Os esquemas de aparência referem-se às generalizações cognitivas acerca do significado, da importância e do possível efeito que a aparência pode ter na vida de um indivíduo (Cash \& Labarge, 1996). Desta forma, o investimento esquemático na aparência é constituído por duas dimensões: a saliência motivacional, que compreende os investimentos para a gestão da aparência, realçando a atratividade; e a saliência auto-avaliativa, que pode ser considerada um tanto disfuncional, uma vez que denota o grau em que o indivíduo considera que a aparência integra a sua identidade e permite aferir o seu valor pessoal, bem como preocupações com a opinião e avaliação de outros acerca da sua aparência (Cash, Phillips, Santos, \& Hrabosky, 2004).

Os níveis elevados de saliência auto-avaliativa traduzem esquemas disfuncionais acerca da imagem corporal, levando a que progressivamente haja uma auto-avaliação negativa da aparência, o que se traduz em níveis mais elevados de insatisfação corporal (Jakatdar, Cash, \& Engle, 2006). A saliência motivacional não se considera patológica desde que os esforços realizados para manter a atratividade não sejam devido a crenças sobre a importância da aparência para o valor próprio (Cash et al., 2004). O estudo de Barbosa et al. (2011), com 690 jovens entre os 15 e 23 anos, com vista a analisar a contribuição diferencial da relação parental e de pares na forma como os jovens experienciam a relação com o seu corpo, salientou a importância da família na construção de uma vivência corporal positiva, revelando-se a variável com mais peso total na imagem corporal. $\mathrm{O}$ estudo revelou também que os jovens com relações de vinculação inseguras com as suas figuras significativas tinham uma relação mais negativa com o seu corpo.

Estando a imagem corporal ligada a aspetos cognitivos e afetivos, salienta-se a importância da autorregulação emocional de forma a atenuar os efeitos que uma discrepância entre o Eu Ideal e o Eu Real possa causar e ainda na conceção positiva da sua imagem corporal (Hughes \& Gullone, 2011). Neste sentido, um individuo que detenha um desenvolvimento emocional adaptativo terá, à partida, um fator protetor face a preocupações com a aparência. Por outro lado, dificuldades na regulação emocional aliada à perceção corporal negativa gerará e manterá emoções e cognições negativas e desajustadas (Muehlenkamp, Peat, 
Claes, \& Smits, 2012), podendo causar sofrimento significativo aos indivíduos e ocasionar um impacto na qualidade de vida e relações interpessoais (Cash \& Fleming, 2002). Uma imagem corporal negativa leva a sentimentos desconfortáveis, como ansiedade, frustração, vergonha e desgosto (Cash, 2008), e por isso a regulação emocional tem um papel essencial neste contexto. Embora a regulação emocional seja um fator importante para a saúde mental, tem recebido pouca atenção, por parte da literatura científica, no que diz respeito às preocupações relativas à imagem corporal (Hughes \& Gullone, 2011). Verifica-se após revisão bibliográfica uma crescente tendência de investigações (eg. Haynos, Roberto, \& Attia, 2015; Lavell, Webb, Zimmer-Gembeck, \& Farrell, 2018) que pretendem analisar o efeito da regulação emocional em perturbações que englobam a imagem corporal (eg. perturbação dismórfica corporal, anorexia, bulimia), mas pouca atenção no que diz respeito a preocupações com a imagem corporal e a sua componente cognitiva e afetiva, como é o caso do investimento na aparência. Desta forma, é relevante investigar em que medida as dificuldades específicas de regulação emocional e também a vinculação aos pais poderão assumir um efeito no investimento esquemático na aparência e ainda aprofundar a componente cognitiva relativa à imagem corporal, devido à escassez de estudos sobre a mesma. Apesar de não se encontrarem, até ao momento, estudos que detenham as dificuldades de regulação emocional como mediadora na associação entre vinculação aos pais e investimento esquemático na aparência, seria interessante analisar o possível efeito nessa associação, ao serem incluídas as dificuldades de regulação emocional, uma vez que as questões ligadas à imagem englobam os níveis físico, emocional e mental (Secchi, Camargo, \& Bertoldo, 2009).

Objetivo

A presente investigação tem como objetivo analisar o papel da vinculação aos pais e da regulação emocional no investimento esquemático na aparência em jovens adultos. Objetiva-se se analisar as diferenças entre a regulação emocional e o investimento esquemático na aparência em função do sexo, idade e satisfação com a imagem corporal. Pretende-se ainda testar o papel mediador das dificuldades de regulação emocional na associação entre vinculação aos pais e o investimento esquemático na aparência.

\section{Método}

\section{Participantes}

Participaram no estudo 948 sujeitos (327 do sexo masculino: 34.5\% e 621 do sexo feminino: $65.5 \%)$ com idades compreendidas entre os 18 e os 30 anos de idade $(M=$ $21.76, D P=3.31)$. Os participantes compreendem habilitações entre o $8^{\circ}$ ano $\left(3^{\circ}\right.$ ciclo $)$ e o ensino superior (mestrado) $(M=12.44, D P=1.03)$. Relativamente à satisfação com a imagem corporal, verifica-se que 761 indivíduos (80.3\%) denotam satisfação, ao passo que $187(19.7 \%)$ referem estar insatisfeitos com a mesma.

\section{Instrumentos}

Foi elaborado um questionário sociodemográfico contendo dados como o sexo, idade e habilitações literárias. Além disso, foi colocada uma questão que remete para a perceção da satisfação corporal. Para avaliar as representações da qualidade de vinculação que os 
sujeitos têm relativamente às suas figuras parentais, optou-se por utilizar o Questionário de Vinculação ao Pai e à Mãe (QVPM; Matos \& Costa, 2001, versão revista). Este é composto por 30 itens, para a mãe e para o pai, distribuídos por 3 dimensões: Inibição da Exploração e Individualidade, com vista a avaliar a perceção de restrições à expressão da individualidade própria, por parte do sujeito; Qualidade do Laço Emocional, pretendendo avaliar a importância da figura parental enquanto figura de vinculação, entendida como única e essencial no desenvolvimento, a quem o sujeito recorre em casos de dificuldades e com quem projeta uma relação duradoura; e a Ansiedade de Separação, que se encontra relacionada com ansiedade e medo de separação da figura de vinculação. Este questionário apresenta uma escala de Likert de 1 a 6 , em que 1 corresponde a "discordo totalmente" e 6 a "concordo totalmente", para cada figura parental. Relativamente às características psicométricas, este instrumento apresenta boa consistência interna nas três dimensões para pai e mãe (entre .81 e .92). Verificaram-se valores de Alpha de Cronbach de .85 para a totalidade do instrumento relativo ao pai e de .79 para a totalidade do instrumento relativo à mãe. A análise fatorial confirmatória revelou valores de ajustamento adequados para pai e mãe, respetivamente: $\chi^{2}(21)=129.55, p=.001 ; \mathrm{CFI}=.98$; TLI $=.96$; RMR $=.06$; RMSEA $=.07$ e $\chi^{2}(21)=122.22, p=.001 ; \mathrm{CFI}=.98 ; \mathrm{TLI}=.96 ; \mathrm{RMR}=.05 ; \mathrm{RMSEA}=.07$.

Para avaliar a regulação emocional, foi utilizada a Escala de Dificuldades de Regulação Emocional - DERS (Gratz \& Roemer, 2004; adaptado para a população portuguesa por Coutinho, Ribeiro, Ferreirinha, \& Dias, 2010). A DERS é uma escala de autorrelato que pretende avaliar dificuldades de regulação emocional clinicamente significativas. É composta por 36 itens e, para além de um coeficiente geral de dificuldade em regular emoções, contém ainda seis dimensões, nomeadamente: Estratégias, onde se observa se existem dificuldades no acesso a estratégias emocionais reguladas; Não-aceitação, que mostra o nível de dificuldade em aceitar as emoções disfóricas e lidar positivamente com elas; Consciência, com a vista a perceber se o sujeito está atento e consciente dos seus estados emocionais; Impulso, que mostra se existem dificuldades em controlar impulsos quando perturbado; Objetivos, que evidencia o nível de dificuldade do sujeito em se envolver em comportamentos centrados em objetivos aquando de uma situação stressante; e Clareza evidenciando dificuldade ao nível da clareza emocional (Coutinho et al., 2010). A cotação é feita numa escala de Likert de 5 pontos que varia de "1- Quase nunca a 5- Quase sempre".

Importa salientar que, na presente amostra, a dimensão consciência não apresentou valores satisfatórios de consistência interna, procedendo-se assim à sua retirada. Após este procedimento, verificou-se um valor de Alpha de Cronbach de .94 para a totalidade do instrumento. Relativamente às dimensões do instrumento, utilizadas no estudo, estas mostram boa consistência interna, onde valor de Alpha de Cronbach varia de .78 a .89. A análise fatorial confirmatória revela um ajustamento adequado $\left(\chi^{2}(32)=136.50, p=.001\right.$; $\mathrm{CFI}=.98 ; \mathrm{TLI}=.97 ; \mathrm{RMR}=.02 ; \mathrm{RMSEA}=.06)$.

Para avaliar o investimento esquemático na aparência foi utilizado o Inventário de Esquemas sobre a Aparência - Revisto - ASI-R (Cash, Melnyk, \& Hrabosky, 2004; adaptado para a população portuguesa por Nazaré, Moreira, \& Canavarro, 2010 ), pretendendo avaliar os níveis de investimento dos indivíduos na sua aparência. Este questionário é composto por 20 itens e 2 dimensões, nomeadamente: Saliência auto-avaliativa, referindo-se às crenças que 
as pessoas têm acerca da forma como o seu aspeto físico permite aferir o seu valor pessoal ou social e o seu sentido de self e, por isso, torna-se uma dimensão mais disfuncional; e Saliencia motivacional, que se refere ao nível de motivação dos indivíduos para gerir o seu aspeto físico e acentuar a sua atratividade. A cotação é feita com base numa escala de Likert com 5 alternativas de resposta que variam entre "Discordo Fortemente" a "Concordo Fortemente".

A partir da análise da consistência interna obteve-se um Alpha de Cronbach de .87 para a totalidade do instrumento. Relativamente às dimensões do mesmo, estas apresentam uma boa consistência interna, onde o valor de Alpha de Cronbach para a dimensão saliência motivacional foi de .79; e para a dimensão saliência auto-avaliativa o valor foi de .85. A análise fatorial confirmatória revelou o ajustamento do modelo $\left(\chi^{2}(5)=21.17, p=.001 ; \mathrm{CFI}=\right.$ $.99 ;$ TLI $=.98 ;$ RMR $=.02 ;$ RMSEA $=.06$ ).

\section{Procedimentos}

Foi realizada uma recolha inicial de 1064 participantes, no entanto após limpeza da base, nomeadamente retirada de outliers e sujeitos com mais de 10\% de missings, restaram 948. A idade foi o critério de recolha, sendo critério de exclusão os jovens com algum tipo de comprometimento cognitivo que os impedisse de responder ao protocolo de investigação. O protocolo foi constituído pelo consentimento informado, seguido dos questionários de autorrelato e o tempo médio de preenchimento foi de aproximadamente 15 minutos. Foi pedida autorização à Comissão de Ética (No 20/2016) da Instituição Universitária que serviu de acolhimento à investigação. A recolha da amostra decorreu presencialmente em contexto de sala de aula em diversos cursos, numa instituição de Ensino Superior da zona Norte de Portugal durante o período de 2 meses, tendo os participantes sido informados sobre a confidencialidade dos dados e voluntariedade da participação.

\section{Resultados}

De modo a proceder à análise das associações entre a vinculação aos pais, dificuldades de regulação emocional e investimento esquemático na aparência, foram realizadas análises correlacionais entre as variáveis supracitadas, através do programa estatístico SPSS - Statistical Package for Social Sciences, na versão 24.0 para o sistema Windows (Tabela 1). Verifica-se a existência de correlações negativas entre a qualidade do laço emocional e clareza, impulso e estratégias (entre $r=-.08$ e $r=-.19, p<.01$ ) para ambas as figuras parentais, no entanto para a figura materna acresce a associação com a não-aceitação $(r=-.07, p<.05)$. As dimensões ansiedade de separação e inibição da exploração e individualidade correlacionam-se positiva e significativamente, de forma fraca e moderada com clareza, impulso, não-aceitação, objetivos e estratégias (entre $r=.12$ e $r=.37, p<.01$ ) para ambas as figuras parentais (Tabela 1). Observa-se ainda que a dimensão ansiedade de separação se correlaciona significativamente de forma positiva com a saliência autoavaliativa e saliência motivacional (entre $r=.13, p<.01$ e $r=.19, p<.01$ ) para ambas as figuras parentais. A dimensão qualidade do laço emocional ao pai e à mãe correlaciona-se positiva e significativamente com a saliência motivacional $(r=.13, p<.01$ e $r=.17, p<$ .01), respetivamente. Já a dimensão inibição da exploração e individualidade relativa ao pai e à mãe, correlaciona-se positiva e significativamente com a saliência auto-avaliativa ( $r=$ $.16, p<.01$ e $\mathrm{r}=.17, p<.01)$, respetivamente $($ Tabela 1$)$. 




Actualidades en Psicología, 33(126), 2019, 97-116 
A saliência auto-avaliativa, por sua vez, correlaciona-se positiva e significativamente de forma fraca e moderada com as dimensões clareza, impulso, não-aceitação, objetivos e estratégias (entre $r=.27$ e $r=.37, p<.01$ ) (Tabela 1).

Variância das dificuldades de regulação emocional e do investimento esquemático na aparência, em função do sexo, idade e satisfação com a imagem corporal dos participantes

De acordo com os objetivos delineados foram realizados test-t para amostras independentes entre as variáveis em estudo, em função da variável sexo e da variável satisfação com a imagem corporal. Para a variável idade, foi realizada uma análise de variância multivariada. Atendendo às dimensões das dificuldades de regulação emocional, face ao sexo, observamse diferenças significativas apenas na dimensão não-aceitação $t(946)=2.28, p=.02$ IC $95 \%[.02, .26]$, onde se verifica que o sexo masculino tem maiores dificuldades em aceitar as emoções disfóricas e lidar positivamente com elas $(M=2.45 ; D P=.86)$, do que o sexo feminino $(M=2.31 ; D P=.93)$ (Tabela 2$)$.

Quanto ao investimento esquemático na aparência, verificam-se diferenças significativas na dimensão saliência auto-avaliativa face ao sexo, $t(725.76)=-3.22, p=.001$ IC 95\% $[-.23,-.06]$. Analisando as médias, observa-se que o sexo feminino apresenta maiores níveis de crenças de como o seu aspeto físico permite aferir o seu valor pessoal $(M=3.15$, $D P=.69)$, do que o sexo masculino $(M=3.01 ; D P=.62)$ (Tabela 2$)$.

Em relação à idade, foram elaborados 3 grupos de acordo com as faixas etárias em que os jovens usualmente experimentam vivências pessoais distintas: o primeiro grupo dos 18 aos 23 anos; o segundo grupo dos 24 aos 27 anos; e o terceiro grupo dos 28 aos 30 anos. Relativamente às dificuldades de regulação emocional face à idade, as análises multivariadas apontam para a existência de diferenças significativas $F(12,1882)=3.18, p=.001, \eta^{2}=.99$. Verifica-se a existência de diferenças na dimensão objetivos $F(2,945)=4.13, p=.02, \eta^{2}$ $=.73$, revelando que o grupo dos 18 aos 23 têm maiores dificuldades no envolvimento de comportamentos centrados em objetivos aquando uma situação ansiogénica $(M=2.88$; $D P=.86)$ do que o grupo dos 28 aos 30 anos $(M=2.63 ; D P=.80)$ (Tabela 3$)$.

Tabela 2

Análise diferencial das dificuldades de regulação emocional e investimento esquemático na aparência em relação ao sexo

Sexo $\quad M \pm D P \quad$ IC95\% $\quad$ Direção das

diferenças

Dificuldades de regulação emocional

Não-aceitação

$\begin{array}{rrrr}\text { Masculino } & 2.45 \pm .86 & {[.02 ; .26]} & 1>2\end{array}$

Investimento esquemático na aparência

Saliência autoavaliativa

$\begin{array}{crrr}\text { Masculino } & 3.01 \pm .62 & {[-.23 ; .06]} & 1<2 \\ \text { Feminino } & 3.15 \pm .69\end{array}$

Actualidades en Psicología, 33(126), 2019, 97-116 
Tabela 3

Análise diferencial das dificuldades de regulação emocional e investimento esquemático na aparência em relação à idade

Idade $\quad \mathrm{M} \pm \mathrm{DP} \quad \mathrm{IC} 95 \% \quad \begin{gathered}\text { Direção das } \\ \text { diferenças }\end{gathered}$

Dificuldades de regulação emocional

Objetivos

$1-18$ aos $23 \quad 2.88 \pm .86 \quad[2.81,2.94]$

$2-24 \operatorname{aos} 27 \quad 2.79 \pm .83 \quad[2.64,2.93]$

$1>3$

$3-28 \operatorname{aos} 30 \quad 2.63 \pm .80 \quad[2.45,2.79]$

Investimento esquemático na aparência

Saliência autoavaliativa

$1-18 \operatorname{aos} 23 \quad 3.14 \pm .70 \quad[3.09,3.18]$

2- 24 aos 27

$2.94 \pm .53 \quad[2.82,3.05]$

$1>2$

3- 28 aos 30

$3.08 \pm .53 \quad[2.94,3.20]$

Saliência motivacional

\begin{tabular}{|c|c|c|}
\hline-18 aos 23 & $3.45 \pm .66$ & {$[3.40,3.50]$} \\
\hline-24 aos 27 & $3.30 \pm .66$ & {$[3.18,3.40]$} \\
\hline 28 aos 3 & $3.43 \pm .63$ & {$[3.29,3.55]$} \\
\hline
\end{tabular}

No que concerne ao investimento esquemático na aparência face à idade, os resultados também apontam para diferenças significativas $F(4,1890)=2.95, p=.02, \eta^{2}=.79$. Atendendo às análises univariadas, observa-se que esta diferenciação ocorre em ambas as dimensões: saliência auto-avaliativa $\left(F(2,945)=5.23, p=.01, \eta^{2}=.83\right)$, denotando que o grupo de indivíduos dos 18 aos 23 anos apresenta maiores níveis de crenças de como o seu aspeto físico permite aferir o seu valor pessoal $(M=3.14 ; D P=.70)$, do que o grupo dos 24 aos 27 anos $(M=2.94 ; D P=.53)$; e saliência motivacional $(F(2,945)=3.22, p=$ $\left..04, \eta^{2}=.62\right)$, verificando-se que o grupo dos 18 aos 23 anos apresenta maiores níveis de atenção à sua aparência, querendo reforçar a atratividade $(M=3.45 ; D P=.66)$, do que o grupo dos 24 aos 27 anos $(M=3.30 ; D P=.66)$ (Tabela 3).

No que diz respeito às dificuldades de regulação emocional face à satisfação com a imagem corporal, verificam-se diferenças significativas em todas as dimensões: clareza $t(241.09)=$ $-5.81, p=.001$ IC 95\% [-.55, -.27]; impulso t(254.65) $=-3.13, p=.001$ IC 95\% [-.37, -.09]; não-aceitação $t(248.89)=-2.54, p=.01 \mathrm{IC} 95 \%$ [-.38, -.05]; objetivos $t(258.56)=-3.14, p$ $=.001$ IC 95\% [-.39, -.09]; e estratégias $t(251.15)=-4.32, p=.001$ IC 95\% [-.45, -.17]. Verifica-se que os indivíduos que se encontram insatisfeitos relativamente à sua imagem corporal apresentam maiores dificuldades de regulação emocional do que aqueles que se encontram satisfeitos, nomeadamente e respetivamente na: clareza emocional $(M=2.57$; $D P=.91$ e $M=2.16 ; D P=.69)$; em controlar impulsos $(M=2.43 ; D P=.92$ e $M=2.21$; $D P=.78)$; em aceitar as emoções $(M=2.53 ; D P=1.07$ e $M=2.32 ; D P=.86)$; em se envolver em comportamentos centrados em objetivos aquando uma situação stressante $(M=3.03 ; D P=.95$ e $M=2.79 ; D P=.82)$; e no acesso a estratégias emocionais reguladas $(M=2.42 ; D P=.91$ e $M=2.11 ; D P=.74)$ (Tabela 4). 
Relativamente às análises diferenciais do investimento esquemático na aparência face à satisfação com a imagem corporal, observam-se diferenças significativas na dimensão saliência auto-avaliativa $t(267.20)=-11.77, p=.001$ IC 95\% [-.74, -.53]. Os indivíduos que se mostram insatisfeitos com a sua imagem corporal revelam níveis de maior atenção com a aparência e crenças de como o seu aspeto físico permite aferir o seu valor pessoal $(M=$ $3.61 ; D P=.67)$, comparativamente com os indivíduos que se encontram satisfeitos $(M=$ 2.98; $D P=.61)$ (Tabela 4).

O papel mediador das dificuldades de regulação emocional na associação entre vinculação aos pais e investimento esquemático na aparência.

De acordo com os resultados obtidos no modelo de equações estruturais através do programa AMOS na versão 23.0, partindo do método de Bootstrap, observa-se um efeito inicial significativo e positivo entre a ansiedade de separação ao pai e a inibição da exploração e individualidade relativa à mãe face à saliência auto-avaliativa $(\beta=.28)$ e $(\beta$ $=.15)$ respetivamente. Constata-se ainda um efeito inicial positivo entre a ansiedade de separação relativa ao pai face à saliência motivacional $(\beta=.30)$ e um efeito negativo entre a ansiedade de separação à mãe e a saliência motivacional $(\beta=-.22)$. Por fim, denotase um efeito inicial positivo entre a qualidade do laço emocional à mãe e a saliência motivacional $(\beta=.28)$.

Tabela 4

Análise diferencial das dificuldades de regulação emocional e investimento esquemático na aparência em relação à satisfação com a imagem corporal

\begin{tabular}{|c|c|c|c|}
\hline $\begin{array}{l}\text { Satisfação com a } \\
\text { imagem corporal }\end{array}$ & $M \pm D P$ & IC95\% & $\begin{array}{c}\text { Direção das } \\
\text { diferenças }\end{array}$ \\
\hline
\end{tabular}

Dificuldades de regulação emocional

\begin{tabular}{|c|c|c|c|c|}
\hline Clareza & $\begin{array}{l}\text { 1- Sim } \\
\text { 2- Não }\end{array}$ & $\begin{array}{l}2.16 \pm .69 \\
2.57 \pm .91\end{array}$ & {$[-.55 ;-.27]$} & $1<2$ \\
\hline Impulso & $\begin{array}{l}\text { 1- Sim } \\
\text { 2- Não }\end{array}$ & $\begin{array}{l}2.21 \pm .78 \\
2.43 \pm .92\end{array}$ & {$[-.37 ;-.09]$} & $1<2$ \\
\hline Não-aceitação & $\begin{array}{l}\text { 1- Sim } \\
\text { 2- Não }\end{array}$ & $\begin{array}{c}2.32 \pm .86 \\
2.53 \pm 1.07\end{array}$ & {$[-.38 ;-.05]$} & $1<2$ \\
\hline Objetivos & $\begin{array}{l}\text { 1- Sim } \\
\text { 2- Não }\end{array}$ & $\begin{array}{l}2.79 \pm .82 \\
3.03 \pm .95\end{array}$ & {$[-.39 ;-.09]$} & $1<2$ \\
\hline Estratégias & $\begin{array}{l}\text { 1- Sim } \\
\text { 2- Não }\end{array}$ & $\begin{array}{l}2.11 \pm .74 \\
2.42 \pm .91\end{array}$ & {$[-.45 ;-.17]$} & 1 \\
\hline
\end{tabular}

Investimento esquemático na aparência

$\begin{array}{lll}\text { Saliência auto-avaliativa } & \text { 1- Sim } & 2.98 \pm .61 \\ & 2-\text { Não } & 3.61 \pm .67\end{array} \quad[-.74 ;-.53] \quad 1<2$


Posteriormente foi analisado o papel das dificuldades de regulação emocional como mediador. Relativamente à figura materna, verifica-se que a qualidade do laço emocional prediz negativamente as dificuldades de regulação emocional $(\beta=-.15)$. Por outro lado, a ansiedade de separação e a inibição da exploração e individualidade predizem positivamente as dificuldades de regulação emocional $(\beta=.24)$ e $(\beta=.27)$, respetivamente. Quanto à figura paterna, não se verificam quaisquer predições face às dificuldades de regulação emocional. Observa-se ainda que as dificuldades de regulação emocional predizem positivamente a saliência auto-avaliativa $(\beta=.41)$.
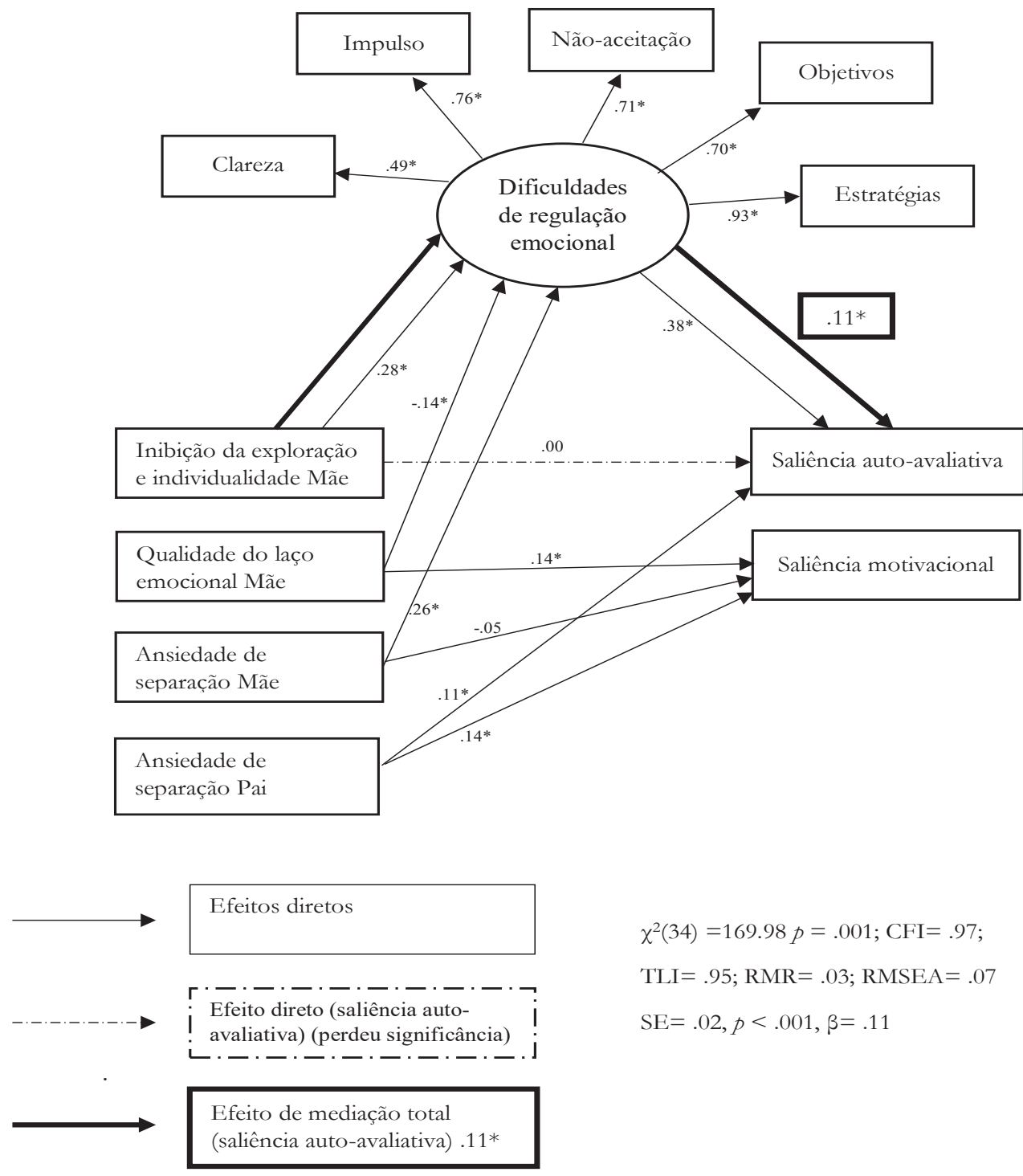

Figura 1. Modelo representativo do efeito mediador das dificuldades de regulação emocional na associação entre inibição da exploração e individualidade mãe e saliência auto-avaliativa. 
Após a realização da testagem do papel mediador das dificuldades de regulação emocional, constata-se que o efeito direito inicial da inibição da exploração e individualidade relativa à figura materna sobre a saliência auto-avaliativa perdeu significância $(\beta=.00)$. Assim, verifica-se uma mediação total com efeito positivo das dificuldades de regulação emocional na associação da inibição da exploração e individualidade relativa à mãe e a saliência autoavaliativa ( $S E=.02 ; \beta=.11$, IC $90 \%[.08, .13])$. No modelo final, observa-se ainda que os efeitos diretos entre a ansiedade de separação ao pai e saliência motivacional, assim como entre a qualidade do laço emocional à mãe e saliência motivacional e ainda entre a ansiedade de separação face ao pai e saliência auto-avaliativa perderam magnitude $(\beta=$ $.14, \beta=.14$ e $\beta=.11$ ), respetivamente. De igual modo, o efeito direto entre a qualidade do laço emocional à mãe e dificuldades de regulação emocional perdeu magnitude $(\beta=-.14)$, ao passo que o efeito direto entre ansiedade de separação face à mãe e dificuldades de regulação emocional ganhou magnitude $(\beta=.26)$. Por fim, o efeito direto entre a ansiedade de separação face à mãe e saliência motivacional perdeu significância $(\beta=-.05)$ (figura 1). Constata-se que os índices de ajustamento do modelo se encontram adequados: $\chi^{2}(34)$ $=169.98 ; p=.001 ; \chi^{2} / G L=4.99 ; \mathrm{CFI}=.97 ; \mathrm{TLI}=.95 ; \mathrm{RMR}=.03 ; \mathrm{RMSEA}=.07$.

\section{Discussão}

O presente estudo teve como principal objetivo analisar o papel da vinculação aos pais e da regulação emocional no investimento esquemático na aparência dos jovens adultos. Observou-se que a qualidade do laço emocional a ambas as figuras parentais se associou de forma negativa com a clareza, impulso e estratégias, e no caso da figura materna acresce a associação com a não-aceitação.

Por outro lado, a ansiedade de separação e a inibição da exploração e individualidade relativa a ambas as figuras parentais associou-se de forma positiva com as dificuldades de regulação emocional na sua totalidade. Deste modo, destaca-se a importância da qualidade do laço emocional aos pais como fator protetor face a dificuldades de regulação emocional nos jovens, evidenciando que uma relação pautada por confiança e segurança parece favorecer a regulação emocional, como o controlo dos impulsos, a clareza e aceitação das emoções, assim como o planeamento de estratégias para regulá-las.

Neste sentido, Bowlby (1973) refere que a qualidade da vinculação desde a infância até à fase adulta, está intrinsecamente ligada ao controlo e maturação emocional dos indivíduos. Em contrapartida, a dependência, insegurança e inibição da individualidade do jovem parecem constituir-se como variáveis que potenciam dificuldades de regulação emocional. Poderá ser explicativo o facto da inibição à exploração e individualidade ser contraproducente, especialmente em jovens adultos, pois interfere no processo de autonomia e desenvolvimento emocional, resultando numa menor capacidade de regulação emocional. Segundo Manzeske e Stright (2009), a jovem adultícia pressupõe que os indivíduos aperfeiçoem, de forma autónoma, as suas próprias estratégias emocionais, com mínimo de interferência das figuras parentais, de forma a consolidarem o processo de autonomia e adaptação. Quando as interações com as mesmas são caracterizadas por controlo excessivo, inconsistência e inibição da exploração, conduzem a sentimentos de desvalorização e menor eficácia na regulação emocional (Greenberg, 1999). 
Relativamente à associação entre vinculação aos pais e o investimento esquemático na aparência, verificou-se que a ansiedade de separação e a inibição da exploração e individualidade relativa a ambas as figuras parentais se associaram positivamente com a saliência auto-avaliativa. Observou-se também que a qualidade do laço emocional e ansiedade de separação a ambas as figuras parentais se associaram positivamente à saliência motivacional. Considera-se que a dimensão ansiedade de separação apenas se assume uma variável negativa quando se experienciam os seus extremos.

Deste modo, a ansiedade pode ser positiva no contexto da vinculação quando exerce um papel adaptativo no desenvolvimento do jovem. Posto isto, níveis considerados normativos de ansiedade de separação, estando intrinsecamente ligados a um ambiente familiar pautado por segurança e confiança, podem potenciar um desenvolvimento ajustado e, por conseguinte, predispõe o jovem a possuir uma imagem de si mais segura e positiva (Barbosa \& Costa, 2001), esforçando-se por manter a sua aparência. Por outro lado, quando a ansiedade de separação se assume extrema e/ ou está presente inibição da exploração e individualidade do jovem, estas variáveis colocam-se como entraves ao processo de autonomia, podendo ter efeitos no desenvolvimento emocional (Ainsworth, 1989; Bowlby, 1988; Manzeske \& Stright, 2009). Este cenário poderá proporcionar ao jovem uma imagem negativa de si, preocupações e insegurança com a sua própria aparência, bem como maior suscetibilidade à opinião alheia, potenciando níveis mais elevados de saliência auto-avaliativa.

No que concerne à associação entre dificuldades de regulação emocional e investimento esquemático na aparência, verificou-se que as dificuldades de regulação emocional (clareza, impulso, objetivos, não-aceitação e estratégias) se associaram positivamente com a saliência auto-avaliativa. As emoções e pensamentos ligados à avaliação e crenças disfuncionais da aparência, se não forem reconhecidos, clarificados, compreendidos e absorverem o jovem por inteiro, ao ponto de o impedir de realizar outras tarefas, acabam por manter distorções cognitivas e reforçar comportamentos desadaptativos (Muehlenkamp et al., 2012), como é o caso da saliência auto-avaliativa.

Por outro lado, os jovens que possuem maiores níveis de aceitação e estratégias emocionais, poderão face a sentimentos disfuncionais relativos à aparência, lidar positivamente com as suas emoções, com vista à modificação de comportamentos e sentimentos que causem o seu desconforto e mantenham a saliência auto-avaliativa elevada. Tendo em conta este aspeto, os jovens com menores dificuldades de regulação emocional, parecem estar mais propensos a investirem de forma mais ajustada na sua aparência.

Face às dificuldades de regulação emocional, verificou-se que o sexo masculino manifesta maiores dificuldades em aceitar as emoções e lidar positivamente com elas. O estudo de Shiovitz Lemonik (2015), realizado com 235 jovens adultos com vista a analisar a desregulação emocional corrobora este resultado, constatando que o sexo masculino evidencia maiores dificuldades a nível da aceitação das emoções. Poderá ser explicativo o facto da expressão emocional, tendo em conta o contexto sociocultural, ser mais restritiva para o sexo masculino, nomeadamente na questão do choro e pela escassa estimulação da verbalização emocional, sendo esperada uma postura mais ativa e racional. Por outro lado, 
as mulheres são mais estimuladas a verbalizar as suas emoções e a lidar com as mesmas, tendo algum suporte das figuras significativas (Núñez, Fernández-Berrocal, Rodríguez, \& Postigo, 2008). Desta forma, os homens poderão inibir as suas emoções, evitando lidar com as mesmas (Carlotto, 2011; Formiga, 2006) e, por conseguinte, acarretar maiores dificuldades em aceitá-las.

No que concerne ao investimento esquemático na aparência face ao sexo, verificou-se que o sexo feminino detém maiores níveis de saliência auto-avaliativa. Tal facto poderá ser explicado pelas maiores preocupações e importância que as mulheres atribuem à sua aparência devido a aspetos socioculturais, que propagam padrões e ideais de beleza mais rígidos para o sexo feminino (Cash et al., 2004). Estas preocupações, o desejo ou exigência de estar à altura do que é imposto socialmente e por vezes a discrepância entre os padrões de beleza e o seu Eu Real, podem originar emoções negativas, insegurança e suscetibilidade à aceitação e opinião de outrem.

Nesta medida, estas variáveis em conjunto, que poderão advir de um contexto familiar marcado por insegurança e falta de confiança, podem culminar num investimento na aparência desajustado, ou seja, em maiores níveis de saliência auto-avaliativa. O estudo de Nazaré et al. (2010), com 226 sujeitos adultos de ambos os sexos, com o objetivo verificar as características psicométricas da versão portuguesa do Inventário de Esquemas sobre a Aparência - Revisto (ASI-R), vai ao encontro deste resultado, evidenciando maiores níveis de investimento na aparência no sexo feminino.

Relativamente às diferenças nas dificuldades de regulação emocional face à idade, os jovens entre os 18 e os 23 anos denotaram maiores dificuldades no envolvimento de comportamentos centrados em objetivos, quando experienciam emoções negativas, do que jovens entre os 28 e os 30 anos. Os jovens mais novos, não tendo adquirido a mesma maturidade, não tendo, à partida, um repertório diversificado de experiências emocionais e vivenciando transformações psicológicas face aos mais velhos, poderão estar mais suscetíveis a experienciarem as suas emoções de forma mais intensa, tendo dificuldades em se concentrarem na realização de tarefas.

Ainda nesta perspetiva, vale salientar que os indivíduos mais jovens, à partida, ainda partilham o mesmo lar com as figuras parentais, tendo estes por sua vez um papel importante no desenvolvimento emocional dos seus filhos. Nesta medida, se o ambiente familiar se colocar como entrave à regulação emocional adaptativa, poderão surgir maiores dificuldades na gestão das emoções (Bowlby, 1969; Manzeske \& Stright, 2009). De acordo com Orgeta (2009), a capacidade de regulação emocional tende a aumentar com a idade, podendo ser devido à elevação da inteligência cristalizada e à diminuição dos níveis de neuroticismo.

Relativamente ao investimento na aparência face à idade, verificou-se que os jovens entre os 18 e 23 anos denotam maiores níveis de saliência auto-avaliativa e de saliência motivacional face aos jovens com idades compreendidas entre 24 e 27 anos. Os indivíduos mais novos parecem esforçar-se mais para manter a sua atratividade e ao mesmo tempo estão mais atentos e preocupados com a sua aparência e opiniões alheias, potenciando o desajuste no investimento na aparência. Os indivíduos mais novos poderão investir mais na sua aparência devido ao início de experiências românticas, que por sua vez implica 
alguma preocupação acerca da avaliação de outras pessoas (Alferes, 2006), o que aliada à imaturidade dos jovens, face aos mais velhos, pode tornar-se disfuncional.

Estes jovens também podem percecionar, valorizar e ter maiores preocupações com o seu corpo, tendo em vista a inserção e aceitação em sociedade (Jager et al., 2017), a qual se encontra recheada de preconceitos e as diferenças culminam em exclusão social e bullying. Assim, o corpo e a aparência poderão ser vistos, nessa idade, como uma ferramenta poderosa para a integração. Consistente com a literatura está um estudo com 443 universitários de ambos os sexos, com intuito de averiguar o pensamento social e comportamentos associados ao corpo, verificando que a aparência possuía um maior peso nas relações interpessoais em indivíduos mais jovens (Camargo, Justo, \& Jodelet, 2010).

No que concerne às diferenças das dificuldades de regulação emocional face à satisfação com a imagem corporal, observou-se que indivíduos insatisfeitos com a mesma denotam maiores dificuldades na clareza emocional, no controlo dos impulsos, na aceitação das emoções, em conseguir realizar tarefas/objetivos aquando de emoções negativas e no acesso a estratégias emocionais reguladas. Os indivíduos insatisfeitos com a sua imagem corporal podem estar propensos a emoções negativas associadas à mesma. Segundo Sim e Zeman (2006), as emoções negativas constituem-se como fatores de risco ao desenvolvimento de insatisfação corporal, e esta, por sua vez, potencia ainda mais emoções negativas devido à contradição entre o corpo real e o ideal. Estas emoções podem tornar-se disfuncionais ao ponto dos indivíduos terem dificuldades em clarificá-las, em se concentrarem em outras tarefas, ou até adotarem estratégias para que possam reverter essa situação.

Relativamente às diferenças no investimento na aparência face à satisfação com a imagem corporal, observou-se que os jovens insatisfeitos com a mesma revelam maiores níveis de saliência auto-avaliativa. Este resultado era expectável devido ao facto dos jovens que se encontram insatisfeitos tenderem a ter maiores preocupações com sua aparência e almejarem estar ao nível dos padrões de beleza socialmente impostos. Indivíduos com elevados níveis de saliência auto-avaliativa têm tendência a distorções cognitivas que, à medida que aumentam, reforçam a insatisfação corporal (Jakatdar et al., 2006).

Mediante a análise do papel mediador das dificuldades de regulação emocional, verificou-se que este parece contribuir positiva e totalmente na associação entre inibição da exploração e individualidade relativa à mãe e saliência auto-avaliativa. Nesta medida, constata-se que a inibição da exploração e individualidade, através de um ambiente familiar pautado por controlo e constituindo-se como entrave ao processo de autonomia, prediz de forma positiva as dificuldades de regulação emocional. Estas, por seu turno, medeiam de forma positiva e total, um investimento potencialmente mais disfuncional na aparência, ou seja, maiores níveis de saliência auto-avaliativa (Muehlenkamp et al., 2012).

\section{Implicacões práticas, limitações e pistas futuras}

A presente investigação auxiliou a compreensão da importância que a qualidade da vinculação aos pais e menores dificuldades de regulação emocional assumem no investimento mais ajustado na aparência dos jovens. Seria relevante a utilização dos dados do estudo para a realização de formação especializada dirigida a técnicos, docentes e 
figuras cuidadoras, como forma de prevenção a comportamentos desadaptativos relativos à aparência em adolescentes e jovens adultos e ainda como consciencialização de que uma relação pautada por segurança, confiança e afeto, constitui-se como fator protetor face ao investimento desajustado na aparência e às dificuldades de regulação emocional.

Salientam-se, contudo, algumas limitações face ao presente estudo, como a disparidade entre sexo, idade e satisfação corporal e a utilização de instrumentos de autorrelato, que aliado à extensão do protocolo, são passíveis de algum cansaço e preenchimento com desejabilidade social. Como pistas futuras, parece relevante a realização de investigações posteriores que assumam um carácter longitudinal, assim como a recolha da amostra ser extensiva a outras áreas do país. Poderia ser conveniente em futuras investigações acerca do investimento na aparência, a inclusão de algumas variáveis como o bem-estar, autoestima, como possíveis fatores protetores e a presença de sintomatologia psicopatológica, devido ao facto da existência de esquemas de aparência desadaptativos poderem provocar sofrimento e originar entraves ao ajustamento psicológico dos indivíduos.

\section{Referências}

Ainsworth, M. (1989). Attachments beyond infancy. American Psychologist, 44(4), 709-716. doi: 10.1037/0003-066X.44.4.709

Alferes, V. R. (2006). Atração interpessoal, sexualidade e relações íntimas. Em J. Vala, \& M. B. Monteiro (Eds.), Psicologia Social (pp. 125-158). Lisboa: Fundação Calouste Gulbenkian.

Barbosa, M. R., \& Costa, M. E. (2001). A influência da vinculação aos pais na imagem corporal de adolescentes e jovens. Cadernos de Consulta Psicológica, 17(18), 83-94.

Barbosa, M. R., Matos, P. M., \& Costa, M. E. (2011). As relações de vinculação e a imagem corporal: Exploração de um modelo. Psicologia: Teoria e Pesquisa, 27(3), 273-282.

Bargh, J. A., \& Williams, L. E. (2007). The nonconscious regulation of emotion. In J. J. Gross (Ed.), Handbook of emotion regulation (pp. 429-445). New York: Guilford Press.

Borelli, J. L., Smiley, P. A., Rasmussen, H. F., Gómez, A., Seaman, L. C., \& Nurmi, E. L. (2017). Interactive effects of attachment and FKBP5 genotype on school-aged children's emotion regulation and depressive symptoms. Behavioural brain research, 325, 278-289. doi: 10.1016/j.bbr.2016.07.035

Bowlby, J. (1969). Attachment and loss. Vol.1: Attachment. New York: Basic Books.

Bowlby, J. (1973). Attachment and loss. Vol.2: Separation, anxiety and anger. New York: Basic Books.

Bowlby, J. (1988). A secure base: Parent-child attachment and healthy human development. London: Basic Books. 
Camargo, B. V., Justo, A. M., \& Jodelet, D. (2010). Normas, representações sociais e práticas corporais. Revista Interamericana de Psicologia, 44(3), 449-457.

Carlotto, M. S. (2011). Tecnoestresse: Diferenças entre homens e mulheres. Revista Psicologia: Organizações e Trabalho, 11(2), 51-64.

Cash, T. F. (2008). The body image workbook: An 8-step program for learning to like your looks (2nd Ed.). Oakland: New Harbinger Publications.

Cash, T. F., \& Fleming, E. C. (2002). The impact of body image experiences: development of the body image quality of life inventory. International Journal of Eating Disorders, 31(4), 455-460. doi:10.1002/ eat.10033

Cash, T. F., \& Labarge, A. S. (1996). Development of the appearance schemas inventory: A new cognitive body-image assessment. Cognitive Therapy and Research, 20(1), 37-50. doi: 10.1007/BF02229242

Cash, T. F., Phillips, K. A., Santos, M. T., \& Hrabosky, J. I. (2004). Measuring “negative body image": Validation of the body image disturbance questionnaire in a nonclinical population. Body Image: An International Journal of Research, 1(4), 363-372. doi: 10.1016/j.bodyim.2004.10.001

Contreras, J., \& Kerns, K. A. (2000). Emotion regulation processes: Explaining links between parent child attachment and peer relationships. In K. A. Kerns, J. M. Contreras, \& A. M. Neal-Barnett (Eds.), Family and peers: Linking two social worlds (pp. 1-25). Westport, CT: Praeger.

Coutinho, J., Ribeiro, E., Ferreirinha, R., \& Dias, P. (2010). Versão portuguesa da Escala de Dificuldades de Regulação Emocional e sua relação com sintomas psicopatológicos. Revista de Psiquiatria Clínica, 37(4), 145-151.

Formiga, N. S. (2006). Diferença de gênero nos antecedentes das emoções de raiva, alegria e tristeza. Revista Científica Eletrônica de Psicologia, 4(6), 1-16.

Gratz, K. L., \& Roemer, L. (2004). Multidimensional assessment of emotion regulation and dysregulation: Development, factor structure, and initial validation of the difficulties in emotion regulation scale. Journal of Psychopathology and Behavioral Assessment, 26(1), 41-54. doi: 10.1023/B:JO BA.0000007455.08539.94

Greenberg, M. T. (1999). Attachment and psychopathology in childhood. In J. Cassidy, \& P. R. Shaver (Eds.), Handbook of attachment: Theory research and clinical applications (pp. 469-495). New York: The Guilford Press.

Gross, J. J., \& Thompson, R. A. (2006). Emotion regulation: Conceptual foundations. In J. J. Gross (Ed.), Handbook of emotion regulation (pp. 3-26). New York: Guilford Press.

Guzmán-González, M. G., Carrasco, N., Figueroa, P., Trabucco, C., \& Vilca, D. (2016). Estilos de apego y dificultades de regulación emocional en estudiantes universitarios. Psykhe, 25(1), 1-13. doi: 10.7764/psykhe.25.1.706 doi:10.7764/psykhe.25.1.706 
Haynos, A. F., Roberto, C. A., \& Attia, E. (2015). Examining the associations between emotion regulation difficulties, anxiety, and eating disorder severity among inpatients with anorexia nervosa. Comprehensive psychiatry, 60, 93-98. doi: 10.1016/j.comppsych.2015.03.004

Hughes, K. E., \& Gullone, E. (2011). Emotion regulation moderates relationships body image concerns and psychological symptomatology. Body Image: An International Journal of Research, 8(3), 224-231. doi: 10.1016/j. bodyim.2011.04.001

Jager, M. E., Comis, D. T., Mucenecki, T. F., Goetz, E. R., Ferraz, R. C., Foletto, J. S., \& Vasconcellos, S. J. L. (2017). O corpo como meio de aceitação e inserção social: Contribuições a partir de Jeffrey Young. Boletim de Psicologia, 67(146), 37-50.

Jakatdar, T. A., Cash, T. F., \& Engle, E. K. (2006). Body-image thought processes: The development and initial validation of the assessment of body-image cognitive distortions. Body Image: An International Journal of Research, 3(4), 325-333. doi: 10.1016/j.bodyim.2006.09.001

Kim, B. R., Stifter, C. A., Philbrook, L. E., \& Teti, D. M. (2014). Infant emotion regulation: Relations to bedtime emotional availability, attachment security, and temperament. Infant Behavior and Development, 37(4), 480-490. doi: 10.1016/j.infbeh.2014.06.006

Lavell, C. H., Webb, H. J., Zimmer-Gembeck, M. J., \& Farrell, L. J. (2018). A prospective study of adolescents' body dysmorphic symptoms: Peer victimization and the direct and protective roles of emotion regulation and mindfulness. Body image, 24, 17-25. doi: 10.1016/j.bodyim.2017.11.006

Malekpour, M. (2007). Effects of attachment on early and later development. The British Journal of Developmental Disabilities, 53(105), 81-95.

Manzeske, D. P., \& Stright, A. D. (2009). Parenting styles and emotion regulation: The role of behavioral and psychological control during young adulthood. Journal of Adult Development, 16(4), 223-229. doi: 10.1007/s10804-009-9068-9

McMillen, J., Katz, C. C., \& Claypool, E. J. (2014). An emotion regulation framework for child welfare intervention and programming. Social Service Review, 88(3), 443-468. doi:10.1086/677656

Morel, K., \& Papouchis, N. (2015). The role of attachment and reflective functioning in emotion regulation. Journal of the American Psychoanalytic Association, 63(4), 15-20. doi: 10.1177/0003065115602447

Moutsiana, C., Fearon, P., Murray, L., Cooper, P., Goodyer, I., Johnstone, T., \& Halligan, S. (2014). Making an effort to feel positive: Insecure attachment in infancy predicts the neural underpinnings of emotion regulation in adulthood. Journal of Child Psychology and Psychiatry, 55(9), 999-1008. doi: $10.1111 /$ jcpp. 12198 
Muehlenkamp, J. J., Peat, C. M., Claes, L., \& Smits, D. (2012). Self-injury and disordered eating: Expressing emotion dysregulation through the body. Suicide and Life-Threatening Behavior, 42(4), 416-425. doi: 10.1111/j.1943278X.2012.00100.x

Nazaré, B., Moreira, H., \& Canavarro, M. C. (2010). Uma perspectiva cognitivocomportamental sobre o investimento esquemático na aparência: Estudos psicométricos do Inventário de Esquemas sobre a Aparência - Revisto (ASI-R). Laboratório de Psicologia, 8(1), 21-36.

Núñez, M. T. S., Fernández-Berrocal, P., Rodríguez, J. M., \& Postigo, J. M. L. (2008). ¿Es la inteligencia emocional una cuestión de género? Socialización de las competencias emocionales en hombres y mujeres y sus implicaciones. Electronic Journal of Research in Educational Psychology, 6(2), 455-474.

Orgeta, V. (2009). Specificity of age differences in emotion regulation. Aging \& Mental Health, 13(6), 818-826. doi: 10.1080/13607860902989661

Park, J., \& Beaudet, M. P. (2007). Eating attitudes and their correlates among Canadian women concerned about their weight. European Eating Disorders Review, 15(4), 311-320. doi: 10.1002/erv.741

Pietromonaco, P. R., \& Barret, L. F. (2000). The internal working models concept: What do we really know about the self in relation to others? Review of General Psychology, 4(2), 155-175. doi: 10.1037/1089-2680.4.2.155

Secchi, K., Camargo, B. V., \& Bertoldo, R. B. (2009). Percepção da imagem corporal e representações sociais do corpo. Psicologia: Teoria e Pesquisa, 25(2), 229-236. doi: 10.1590/S0102-37722009000200011 